\title{
ATLAS jet and missing energy reconstruction, callibration and performance in LHC Run-2
}

\section{Jets}

Collimated sprays of hadrons are the dominant physics objects arising in LHC collisions. Jets are playing a key role in many Standard Model (SM) physics analyses and searches of new phenomena, e.g. ATLAS jets measurements provide a valuable test of QCD in the multi-TeV regime

\section{Jet reconstruction and calibration} Jets are reconstructed in ATLAS with the anti- $\mathrm{k}_{\mathrm{t}}$ jet algorithm, taking topologically connected calorimeter cells, called clusters, as the input, exploiting its main advantage of effective noise suppression. The jet energy scale (JES) calibration brings measured jet energies to that of particle level. JES calibration is implemented as the step by step correction procedure taking the inputs from various detectors.

\section{Origin correction}

Corrects the jet to point to the primary event vertex instead of the geometrical center of ATLAS detector
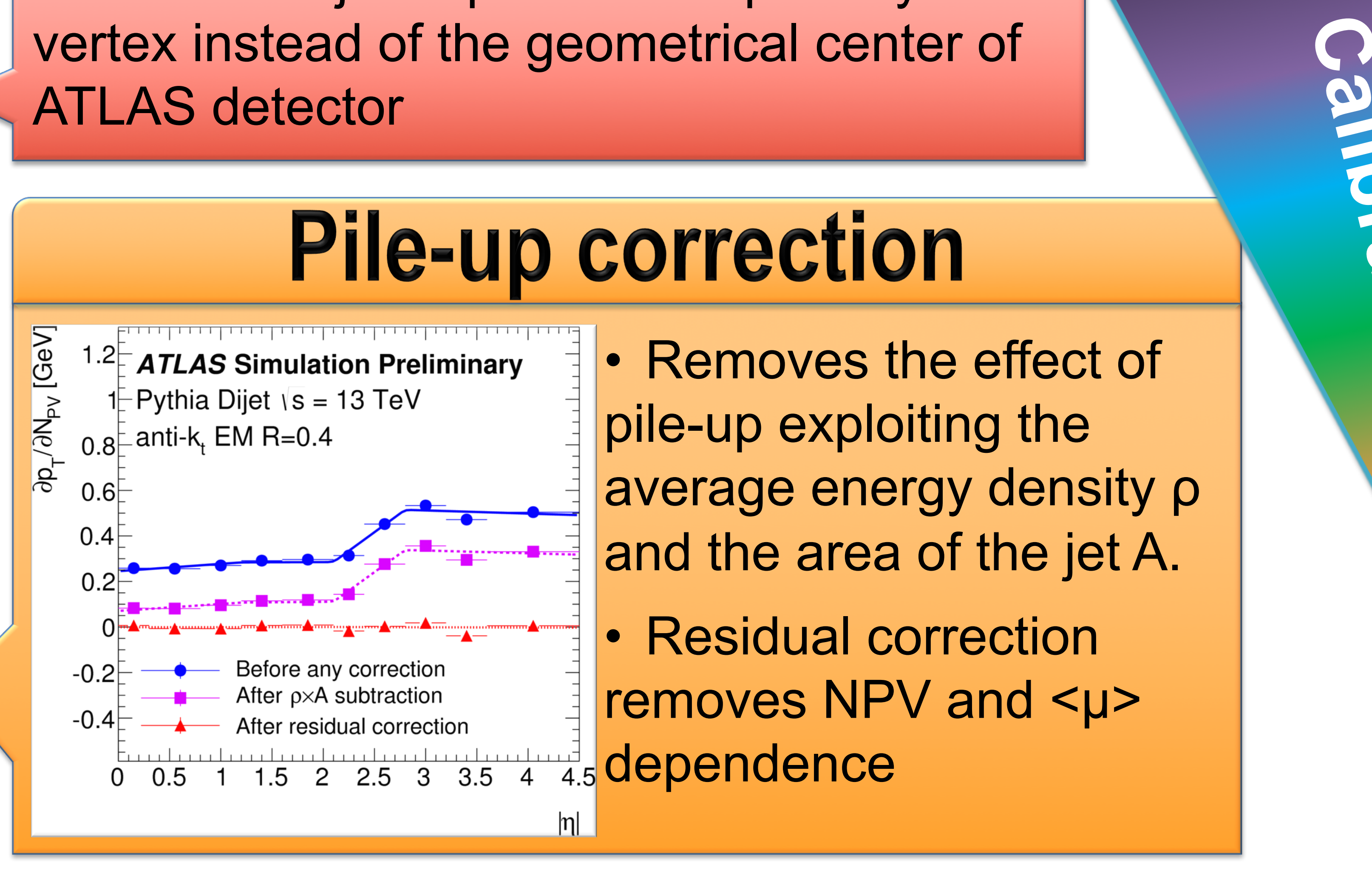

MC JES and $\eta$ correction

- Corrects jet energy for the relative reco-truth difference of calorimeter energy response in

simulation

- Corrects $\eta$ direction of the jet in specific detector regions where the jet $\eta$ bias is observed

\section{Global sequential correction}

Reduces jet response dependence on the flavour of the initiated-jet parton. Flattens the jet response dependence on the: 1. energy fraction in the last electromagnetic calorimeter layer

2. energy fraction in the first hadronic calorimeter layer

3. number of tracks

4. track width ( width $_{\text {trk }}$ )

5. number of muon segments behind jet

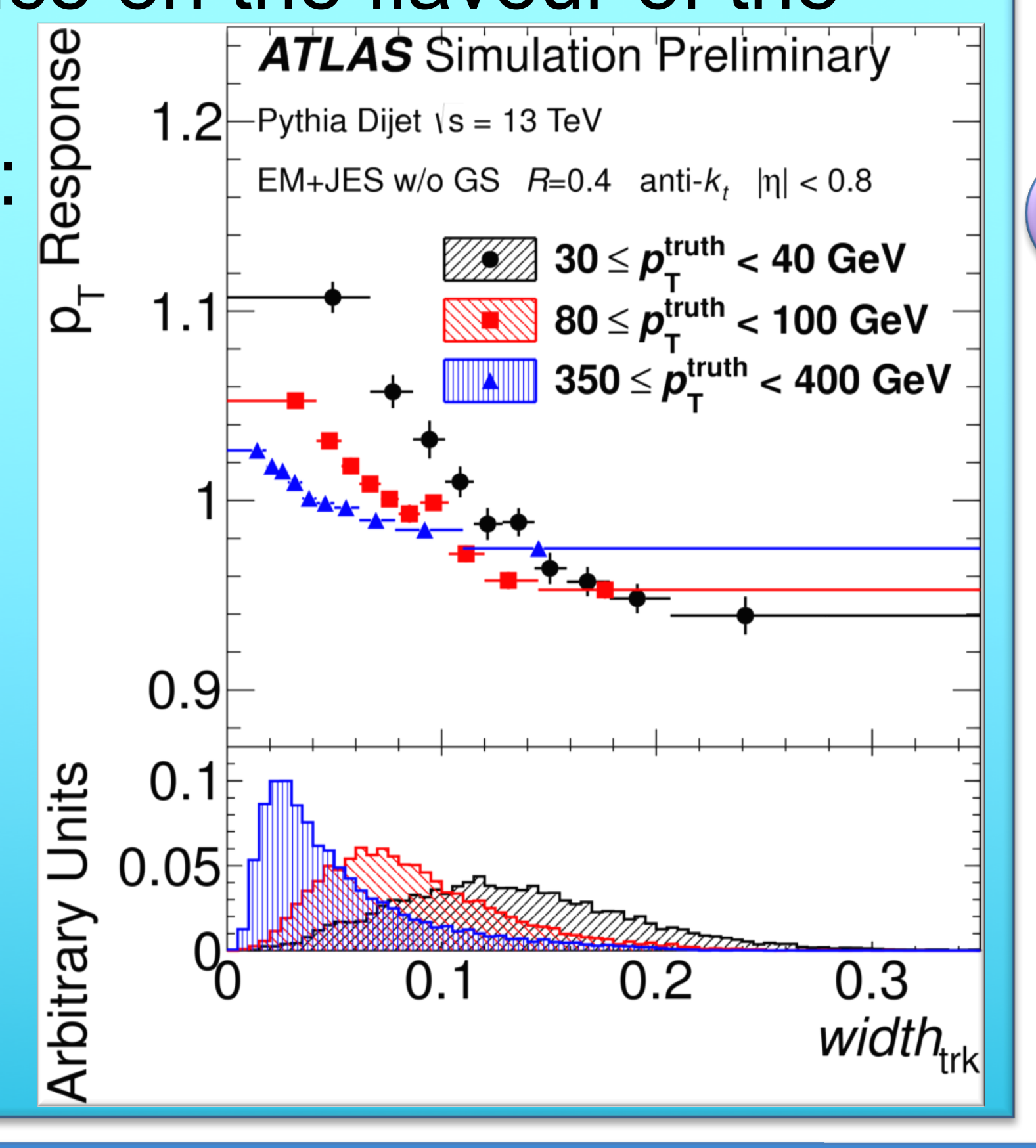

Missing energy $\left(\mathrm{E}_{\mathrm{T}}^{\mathrm{miss}}\right) \quad i \overrightarrow{\mathrm{B}}$

Missing energy is the measure of transverse momentum imbalance created by the detected and well measured objects in an event

$\mathrm{E}_{\mathrm{T}}^{\text {miss }}=-\sum \mathrm{p}_{\mathrm{T}}^{\mathrm{e}}-\sum \mathrm{p}_{\mathrm{T}}^{\gamma}-\sum \mathrm{p}_{\mathrm{T}}^{\tau}-\sum \mathrm{p}_{\mathrm{T}}^{\mu}-\sum \mathrm{p}_{\mathrm{T}}^{\text {jet }}-\sum \mathrm{p}_{\mathrm{T}}^{\text {soft }}$

The missing energy performance drives

SUSY and dark mater searches in LHC.

- Reconstruction and performance is

estimated in events with small $\mathrm{E}_{\mathrm{T}}^{\mathrm{mi}}$

such as $Z \rightarrow(e e / \mu \mu), W \rightarrow(e v / \mu \nu)$ and $t \bar{t}$.

- The soft term ( $p_{T}^{\text {soft }}$ ) in ATLAS is built

from tracks not associated to any

reconstructed hard object $\rightarrow$ Track

Soft Term (TST) $\mathrm{E}_{\mathrm{T}}^{\text {miss }}$

- TST $\mathrm{E}_{\mathrm{T}}^{\mathrm{miss}}$ is insensitive

to pile-up, but missing

contributions from soft

neutral particles.

- The Run-2 brand new technique for suppressing forward pile-up jets (fJVT) improves $E_{\mathrm{T}}^{\text {miss }}$ resolution in high pile-up conditions.

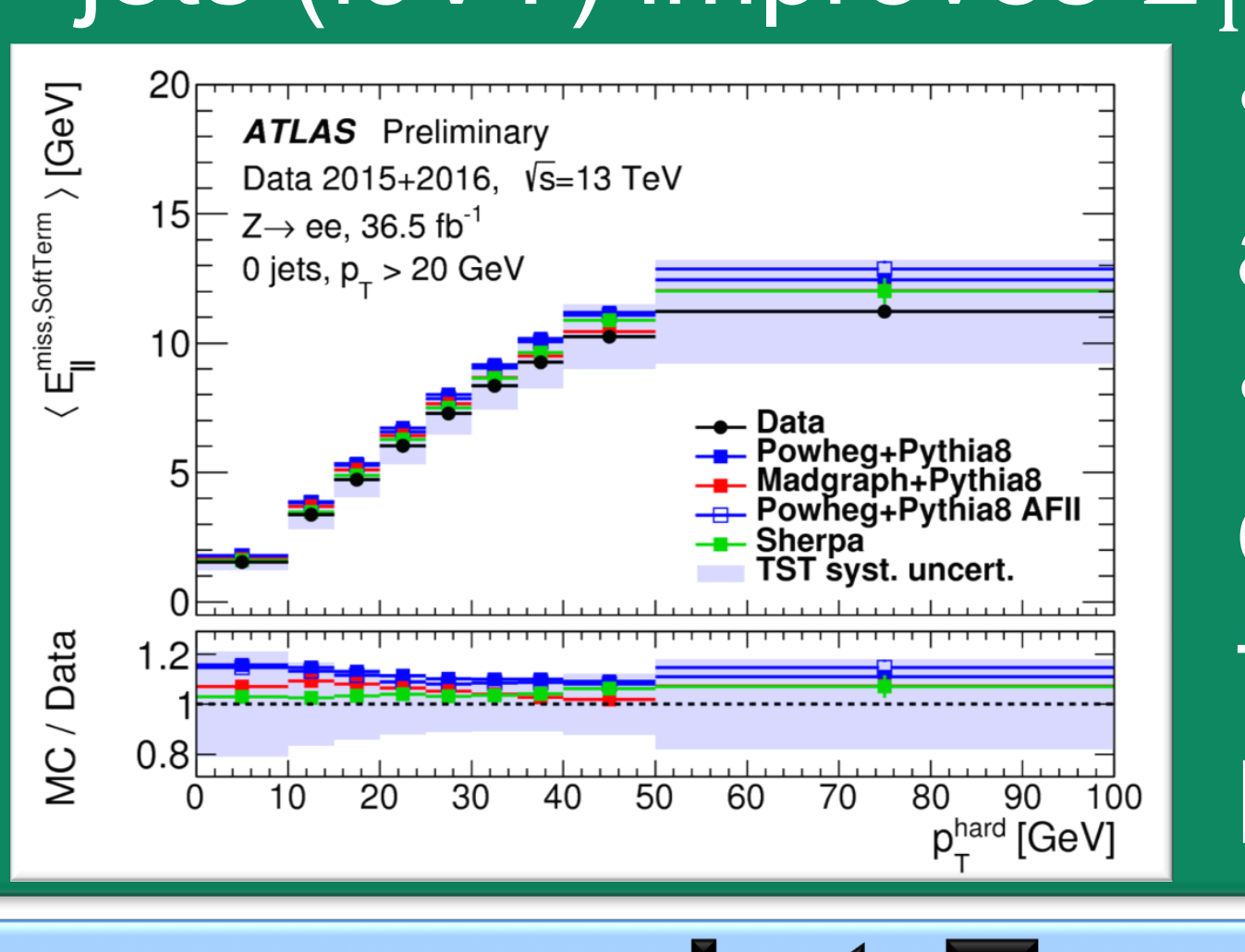
- The hard object systematics uncertainties are propagated to $\mathrm{E}_{\mathrm{T}}^{\mathrm{m}}$

- The balance of the soft term with the calibrated physics objects is used to estimate the soft-term systematic uncertainties by studying data and MC discrepancies.

\section{Jet Energy Resolution (JER)}

The JER is parametrised with the function:

$$
\frac{\sigma\left(p_{T}\right)}{p_{T}}=\frac{N}{p_{T}} \oplus \frac{S}{\sqrt{p_{T}}} \oplus C
$$

The noise $(N)$, stochastic $(S)$ and constant $(C)$ terms were measured in situ with $Z / \gamma+j e t s$ and dijet events.

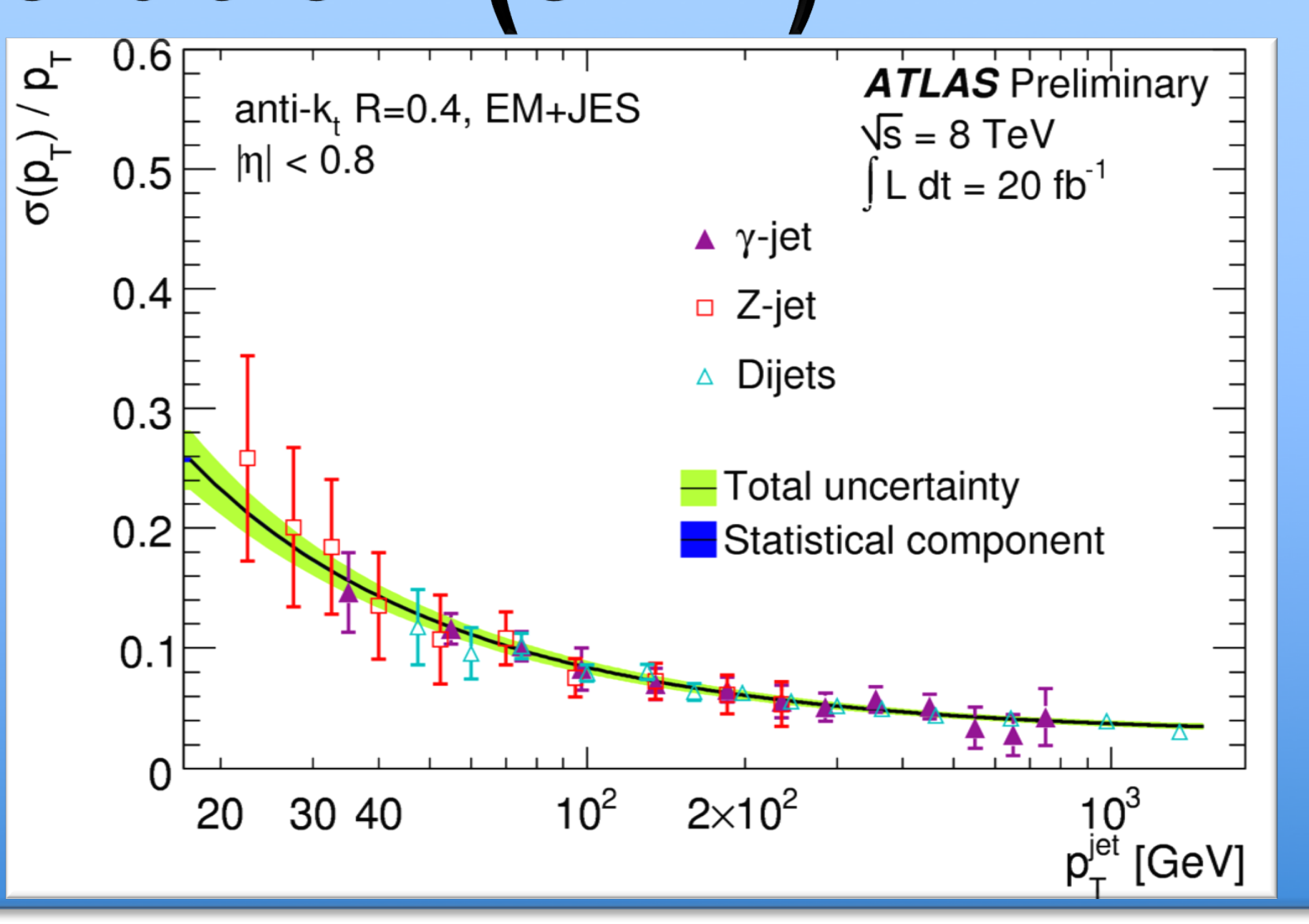

\section{JES Systematic uncertainties}

The jet calibration is supplied with the set of 88 independent sources of systematics uncertainties to correctly account for all correlations in jet calibration.

A reduced set of systematic uncertainties is produced to simplify physics analyses while keeping the loss of correlation information to a minimum.

\section{In situ residual correction}

Corrects the jet $\mathrm{pT}$ in data using a well calibrated reference object in dijet, Z/ $\gamma$ and multijet events - $\eta$-intercalibration exploits central jets to calibrate forward jets

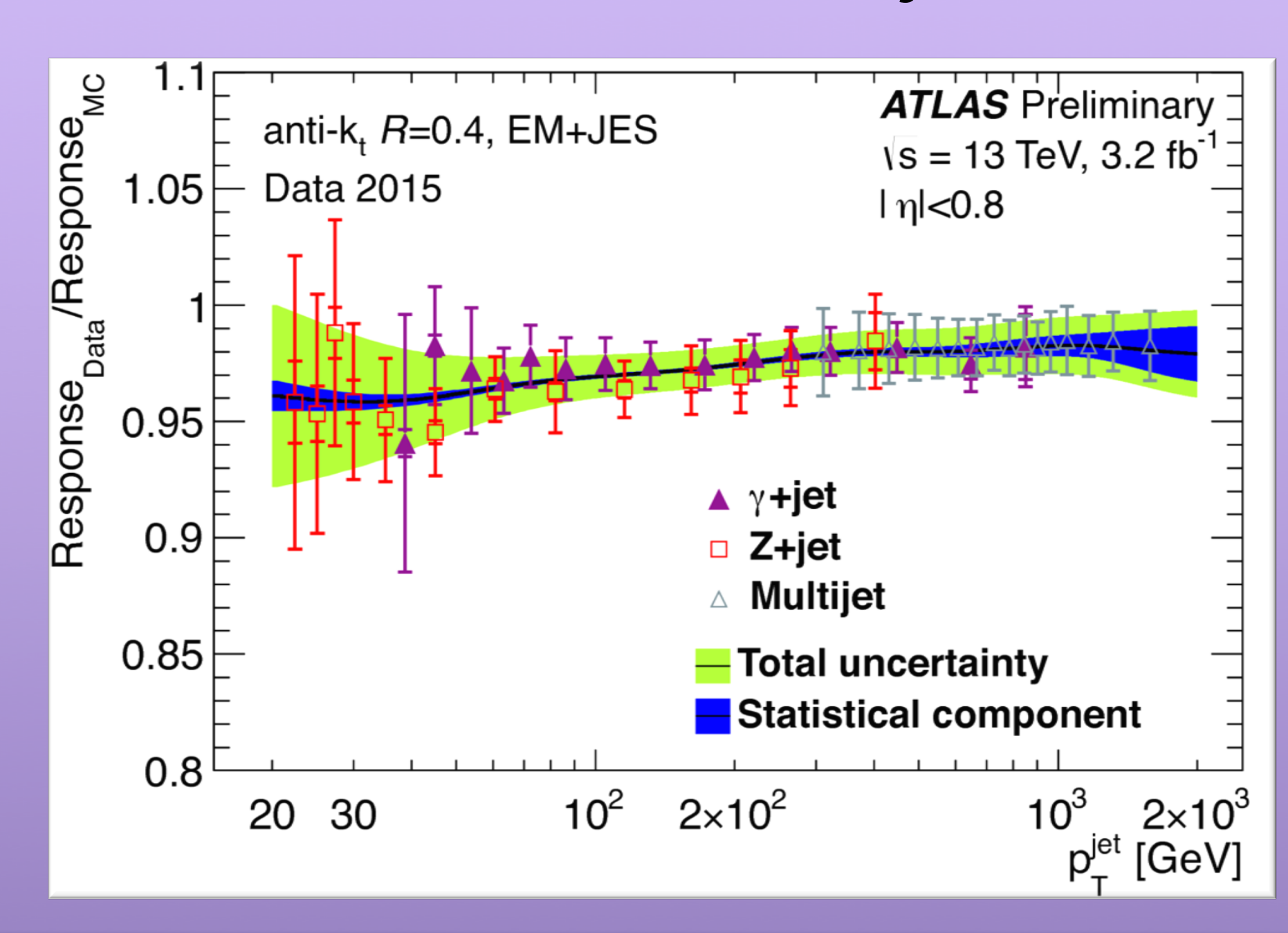

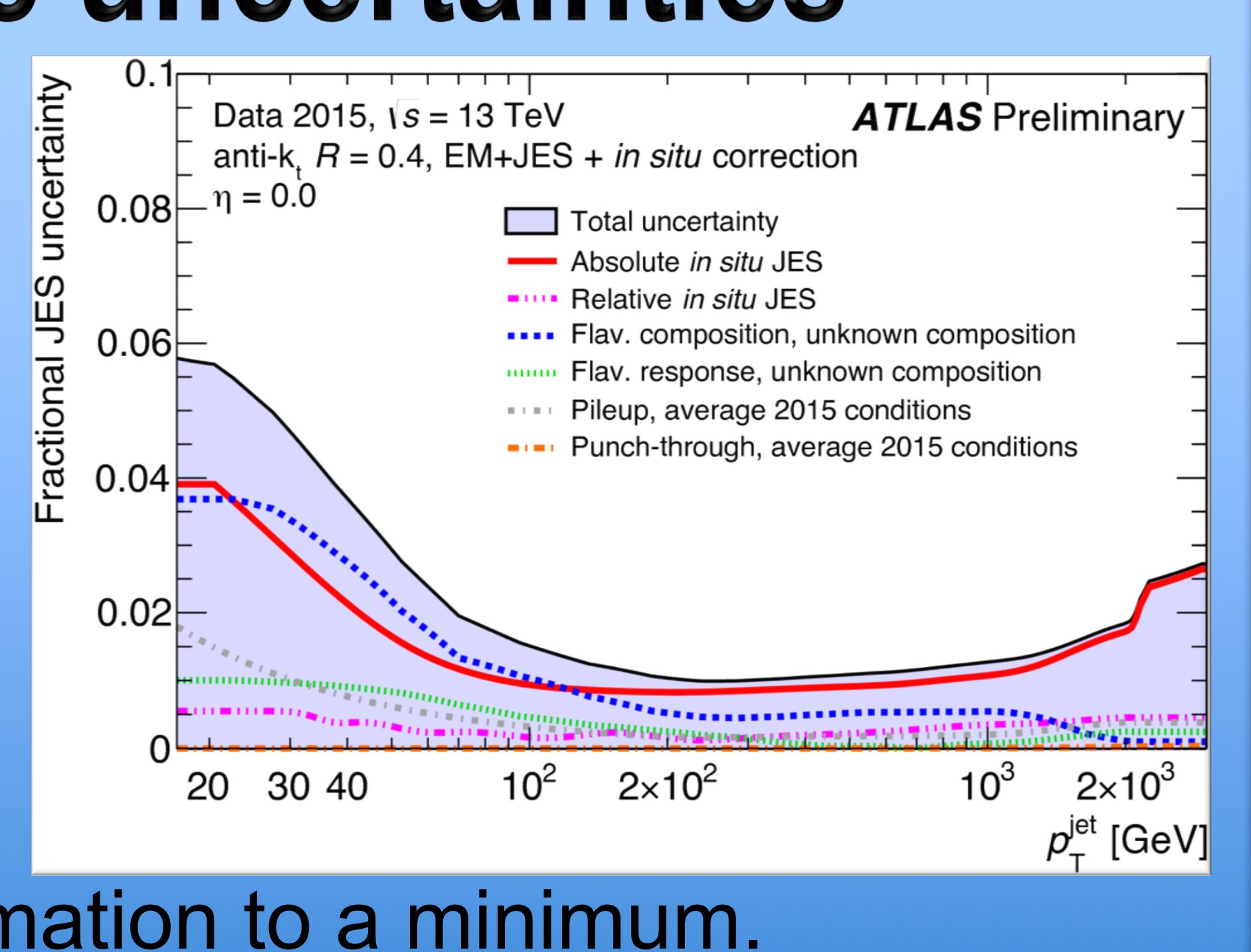

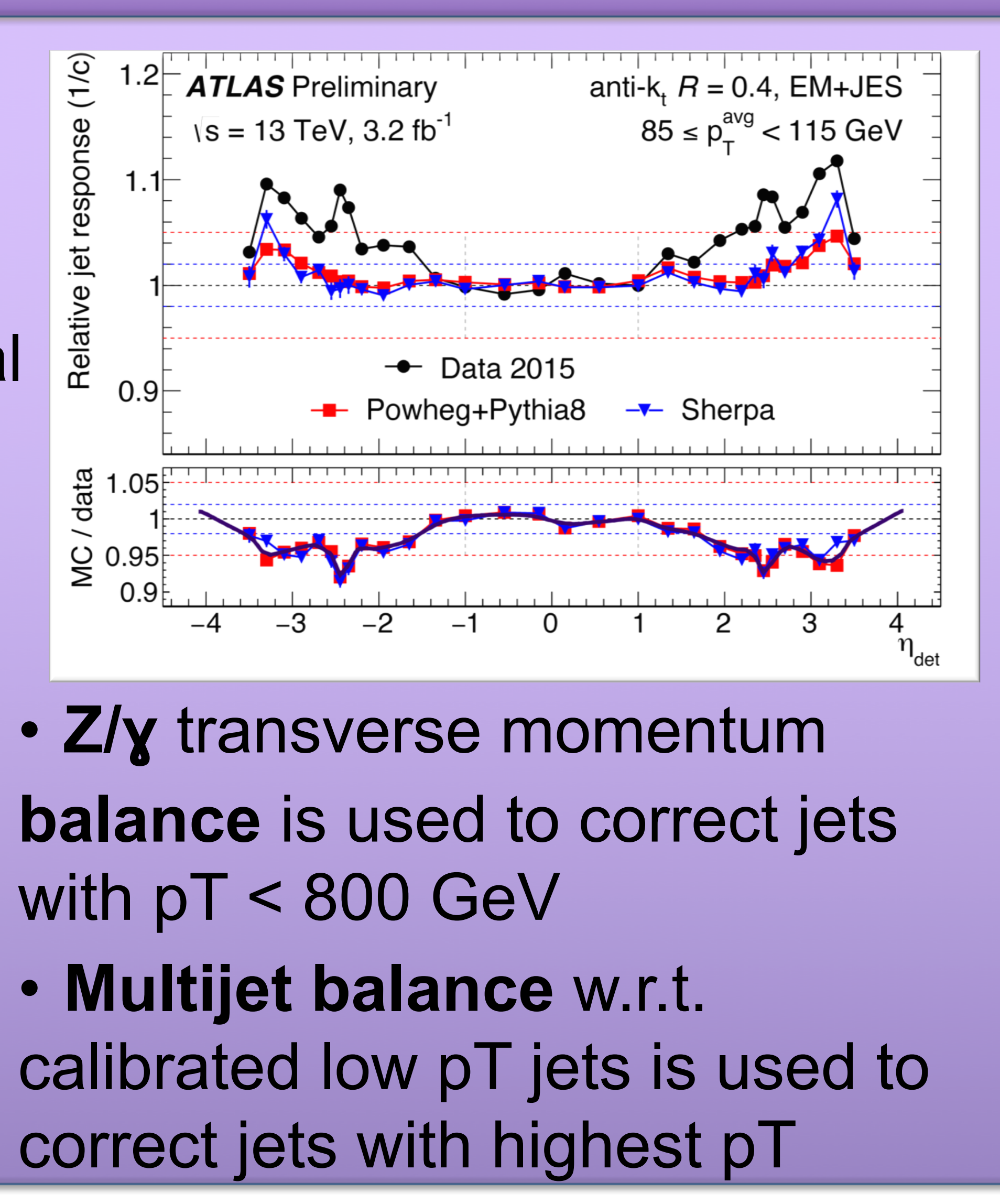

